\title{
Quantifying the PR Interval Pattern During Dynamic Exercise and Recovery
}

\author{
Aline Cabasson*, Student Member, IEEE, Olivier Meste, Member, IEEE, Grégory Blain, and Stéphane Bermon
}

\begin{abstract}
We present a novel analysis tool for time delay estimation in electrocardiographic signal processing. This tool enhances PR interval estimation (index of the atrioventricular conduction time) by limiting the distortion effect of the $T$ wave overlapping the $P$ wave at high heart rates. Our approach consists of modeling the $T$ wave, canceling its influence, and finally estimating the $P R$ intervals during exercise and recovery with the proposed generalized Woody method. Different models of the $T$ wave are presented and compared in a statistical summary that quantitatively justifies the improvements introduced by this study. Among the different models tested, we found that a piecewise linear function significantly reduces the $T$ wave-induced bias in the estimation process. Combining this modeling with the proposed time delay estimation method leads to accurate PR interval estimation. Using this method on real ECGs recorded during exercise and its recovery, we found: 1) that the slopes of PR interval series in the early recovery phase are dependent on the subjects' training status (average of the slopes for sedentary men $=0.11 \mathrm{~ms} / \mathrm{s}$, and for athlete $\mathrm{men}=0.28 \mathrm{~ms} / \mathrm{s}$ ), and 2) an hysteresis phenomenon exists in the relation PR/RR intervals when data from exercise and recovery are compared.
\end{abstract}

Index Terms-Electrocardiography (ECG), exercise, maximum likelihood estimation, PR interval, PR/RR hysteresis, time delay estimation.

\section{INTRODUCTION}

$\mathbf{E}$ STIMATING variability of the heart period series during exercise and recovery is a real challenge in biomedical engineering. One reason is that the global understanding of the interaction between the neural activity and the cardiac outputs is relevant to improving performances of future pacemakers, and to enriching knowledge for medical diagnosis. For instance, the analysis of the PR interval pattern could be performed in order to evaluate not only the sympathetic-parasympathetic balance, but also to reveal the atrioventricular conduction properties [1].

The analysis of PR intervals during exercise and recovery has been rarely addressed to date [2], [3]. The main reason is that the estimation of these intervals is particularly difficult at high heart rates because $\mathrm{T}$ wave tends to overlap the $\mathrm{P}$ one, which biases $\mathrm{P}$

Manuscript received March 11, 2009; revised June 1, 2009 and June 29, 2009. First published August 18, 2009; current version published October 16, 2009. Asterisk indicates corresponding author.

${ }^{*}$ A. Cabasson is with the Laboratory I3S, Centre National de la Recherche Scientifique, University of Nice, Sophia Antipolis 06903, France (e-mail: cabasson@i3s.unice.fr).

O. Meste is with the Laboratory I3S, Centre National de la Recherche Scientifique, University of Nice, Sophia Antipolis 06903, France (e-mail: meste@i3s.unice.fr)

G. Blain is with the Laboratory of Physiological Adaptations, Motor Performance and Health, Faculty of Sports Sciences, Nice 06205, France (e-mail: blain@unice.fr).

S. Bermon is with the Institute of Sports Medicine and Surgery, Monte Carlo 98000, Monaco (e-mail: bermons@im2s.mc).

Digital Object Identifier 10.1109/TBME.2009.2028694

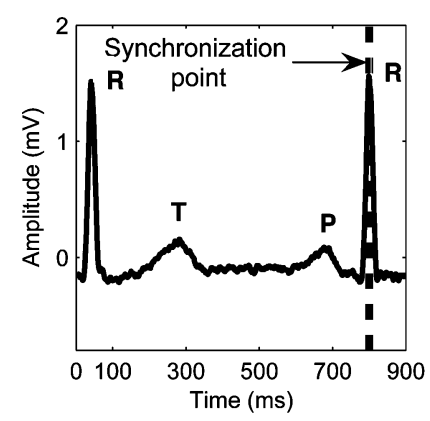

(a)

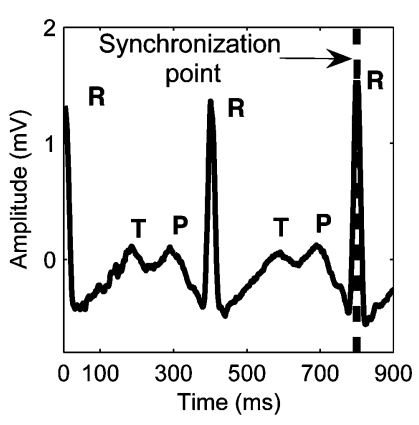

(b)
Fig. 1. Example of (a) a real ECG where the $\mathrm{T}$ and $\mathrm{P}$ waves are disjointed at rest and (b) overlapped during exercise.

wave occurrence detection (see Fig. 1). Consequently, new ad hoc time delay estimators have to be designed. In this paper, we focus on time delay estimation (TDE) in electrocardiographic signals on healthy subjects.

Among the well-known time delay estimators, some techniques have been proposed in different areas of biomedical processing to solve TDE problems for noisy and unknown signals. Cross-correlation approaches, which are based on the detection of the maximum of the cross-correlation, are the most frequently used for TDE [4], [5]. Similar problems are solved using the generalized cross-correlation technique [6], [7]. In particular, the maximum likelihood (ML) TDE has been shown to provide an unbiased time delay estimate under ideal conditions, i.e., without shape or time scale changes of the signals of interest [8]. When the signal under consideration is unknown, i.e., the $\mathrm{P}$ wave in our case, Woody's method [9] is a good candidate that belongs to the cross-correlation family. Besides, the Improved Woody method outperforms significantly Woody's technique [10]. Moreover, for TDE problems, several other techniques have been used mainly working in the frequency domain [11]-[13]. Unfortunately, the introduction of an a priori concerning the overlapping $\mathrm{T}$ wave is tedious in the frequency domain. Alternatively, the monophasic behavior can be well described in the time domain. Also, the wavelet transform is a good candidate for bioelectrical signal analysis and interval estimation [14]. While it has been shown to be a promising tool, these typical methods do not overcome the overlapping problem because the $\mathrm{T}$ and the $\mathrm{P}$ waves share the same frequency band. This drawback will be illustrated in the following. Even so, the wavelet transform applied to ECG characterization could be seen as a derivative function. The zero-crossing locations provide the maximum of the wave of interest. Instead of using this transform, the derivative of the signal can be directly estimated, 
even in presence of noise [15], [16]. While this application is attractive, it can not deal with the problem of overlapping.

Therefore, in this paper an original analysis tool which improves significantly the estimation of the PR intervals during rest, exercise and recovery is proposed. This method combines: 1) a generalized version of the Woody technique using an iterative ML estimator (MLE) and 2) efficient modelings of the T wave which overlaps the $\mathrm{P}$ wave. The remainder of the paper is organized as follows. Section II deals with the proposed Generalized Woody method where the observation model accounts for an additional interfering wave. In Section III, different models for the $\mathrm{T}$ wave are presented: a constrained polynomial function or a constrained piecewise linear function. In Section IV, performance of the different methods are evaluated using synthetic data. This analysis is supplemented by findings from real ECG data. Then, the obtained results are discussed in Section V. Finally, a conclusion is made and some perspectives are suggested in Section VI.

\section{Generalized Woody Method}

During high-intensity exercise, the $\mathrm{P}$ wave onset measurement is not reliable because of the superimposed $T$ wave (see Fig. 1), and the $\mathrm{P}$ wave peak location could be influenced by shape changing, as mentioned in [17]. In addition to the effect of noise, it is clear that the use of these fiducial points will be biased. While the shape of the $\mathrm{P}$ wave is subject to changes during exercise, its global width is almost invariant [18]. This property will permit one to use the correlation-based method, as will be shown in the following. The aim is to reduce the bias of this time delay estimation since the variability of this interval between exercise and recovery is about $10 \mathrm{~ms}$ for a same given heart period (see Fig. 8) [4], [5].

Woody [9] presented a system that is based on iterative correlation-averaging techniques. Later, Pham et al. studied the estimation of variable latencies of noisy signals [12]. Jaśkowski and Verleger [13] referred to a more general model in which the amplitude variability defined by the coefficient $\alpha_{i}$ is also allowed

$$
x_{i}(n)=\alpha_{i} s_{d_{i}}(n)+e_{i}(n)
$$

where, in our case, $s$ is the $\mathrm{P}$ wave.

However, this observation model is too simple for studying the $\mathrm{P}$ wave during exercise. In the signal model $(1), x_{i}(n)$ represents the amplitude of the $i$ th observation at time sample $n(0<$ $n<N$, where $N$ denotes the number of data samples for each trial, and $i=1, \ldots, I$, where $I$ is the number of trial). Each observation contains an unknown transient template wave $s(n)$, delayed by $d_{i}$, as $s_{d_{i}}(n)=s\left(n-d_{i}\right)$. In this simple case, the only interfering component is the observation random noise $e_{i}(n)$, which follows a normal distribution law $\mathcal{N}\left(0, \sigma^{2}\right)$.

As in exercise tests the observation segment includes the $\mathrm{T}$ wave, this interfering wave can be added to our global observation model as a function $\mathbf{f}\left(\boldsymbol{\theta}_{i}\right)$ linearly parameterized. We assume that the $\mathrm{T}$ wave should be described by a regular and smooth function, for instance an $L$ th order polynomial function, or by a piecewise defined function. Note that different models are described in Section III.
Finally, our global observation model is expressed as

$$
x_{i}(n)=\alpha_{i} s_{d_{i}}(n)+f\left(n ; \boldsymbol{\theta}_{i}\right)+e_{i}(n)
$$

where $i, i=1, \ldots, I$, is the index of the trial, $\alpha_{i}$ is a positive coefficient, and the variable $d_{i}$ is the $i$ th PR interval to be estimated up to a constant. It is clear that $s$ is not uniquely defined since shifting $s$ leads to shift in an opposite direction all the $d_{i}$ s values. This lack of identifiability will be solved by imposing the average of the estimated delays to be equal to a constant (e.g., 0).

In order to estimate the delays $d_{i}$, the iterative MLE is used. The details of the demonstration are reported in the Appendix. In summary, the criterion to be minimized is $J$

$$
J=\sum_{i=1}^{I}\left\|\mathbf{x}_{i}-\mathbf{f}\left(\boldsymbol{\theta}_{i}\right)-\frac{\alpha_{i}}{I} \sum_{k=1}^{I} \frac{1}{\alpha_{k}}\left(\mathbf{x}_{k, d_{i}-d_{k}}-\mathbf{f}_{d_{i}-d_{k}}\left(\boldsymbol{\theta}_{k}\right)\right)\right\|^{2} .
$$

Note that the third term in (3) is the average of the synchronized observations where the corresponding $\mathbf{f}_{d_{i}}$ has been subtracted. Thanks to the development given in the Appendix in Section VII, the criterion $J$ can be minimized in an iterative way. This corresponding algorithm starts by replacing the third term by the average of the original observations. For the next step, the third term will be updated using the estimated parameters. For each step, i.e., each $i, d_{i}$ is chosen in a given expected range in order to shift the average. Thus, thanks to linearity, $\alpha_{i}$ and $\boldsymbol{\theta}_{i}$ are given by the least square (LS) estimator, and the corresponding $J_{i}\left(d_{i}\right)$ are computed. Finally, the estimated $\widehat{d_{i}}$ corresponds to the minimum value of $J_{i}\left(d_{i}\right)$. For the next step, a new template is computed using the estimated parameters. If necessary, the process can be iterated depending on the convergence of the algorithm. After convergence, the algorithm produces estimated $\widehat{d}_{i}$ : the estimated PR intervals up to a constant.

From a theoretical point of view, the global modeling of the observations will involve the unknown delays in a nonlinear manner. Unlike the similar models introduced in [12] and [13], we will assume that a ML estimator exists. In [11], [19], it has been shown that, when this identification model is addressed with an ML approach, saddle point singularities appear. Thus, this theoretical assessment will not be in the scope of this paper.

The following section presents different models of the function $\mathbf{f}\left(\boldsymbol{\theta}_{i}\right)$ that is the overlapping $\mathrm{T}$ wave. In order to reduce the bias in the estimation, we take into account the $\mathrm{T}$ wave properties, such as its monophasic shape. This will lead to a constrained solution where $\mathbf{f}\left(\boldsymbol{\theta}_{i}\right)$ will be supposed to be strictly decreasing in the observation window. According to this decreasing constraint, the estimation method based on an iterative ML technique appears to be a sum of LS problem with linearinequality constraint (LSI problem), that will be converted in a least distance programming (LDP) problem [20].

\section{Modeling OF OverLAPPING T WAVE}

In this section, different models of the $\mathrm{T}$ wave are proposed. For instance, a template could be optimally segmented from a real $\mathrm{T}$ wave in the resting period. Unfortunately, the shape of the $\mathrm{T}$ wave varies as long as the effort increases, discarding this 


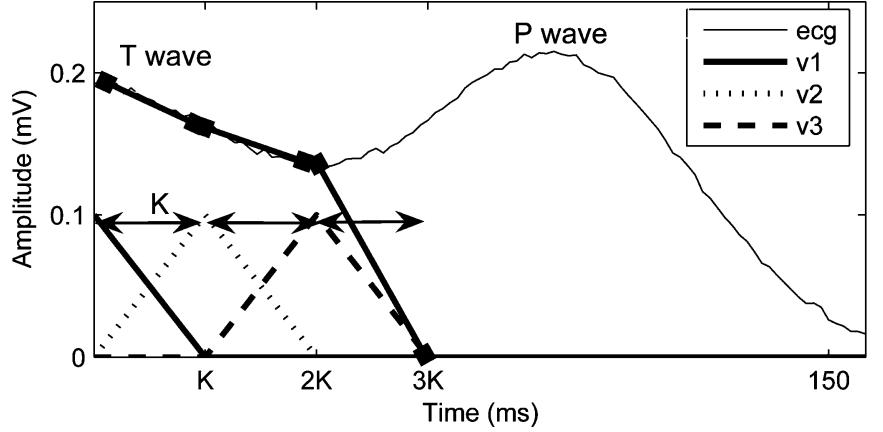

Fig. 2. Example of a real $\mathrm{T}$ wave modeled as a decreasing piecewise linear function based on three functions $\mathbf{v}_{l}\left(M_{\mathrm{P}-3}\right)$. The piecewise function corresponds to the estimated $\mathrm{T}$ wave by the proposed method using the vectors $v_{1}$, $v_{2}$, and $v_{3}$ in a real case.

simple approach. Moreover, methods based on spline interpolation can not be applied because the anchor points of the $\mathrm{T}$ wave are hidden when the fusion occurs.

The outline of our PR intervals estimator algorithm is:

1) modeling of the decreasing part of the $T$ wave imposing additional constraints; these constraints will account for the decreasing behavior of the ending part of the $\mathrm{T}$ wave (see Fig. 2);

2) adaptation of the $T$ wave model to our global observation model presented in Section II;

3) estimation of the PR intervals using an iterative ML technique which includes Least Square solution with linear inequality constraints.

We recall that we consider a model for the observations which is defined by the equation (2) where the variable $d_{i}$ is the $i$ th PR interval to be estimated up to a constant and the decreasing part of the $\mathrm{T}$ wave is represented by a function $\mathbf{f}\left(\boldsymbol{\theta}_{i}\right)$ linearly parameterized. Note that the function $\mathbf{f}\left(\boldsymbol{\theta}_{i}\right)$ is dependent to $i$ in order to allow the shape variation of the $\mathrm{T}$ wave.

\section{A. Model Using Lth Order Polynomial Function $\left(M_{L}\right)$}

We assume that the decreasing part of the $T$ wave is described by a regular and smooth function, i.e., an $L$ th order polynomial function characterized by its coefficients in the vector $\boldsymbol{\theta}_{i}$

$$
f\left(n ; \boldsymbol{\theta}_{i}\right)=\sum_{l=0}^{L} \theta_{i}[l] n^{l} .
$$

In a previous work [21], we considered that $L=1$, i.e., we take into account the $\mathrm{T}$ wave modeled as a straight line $\left(M_{1}\right)$. This method have been tested for a 2 nd order but we will only present in this work the model corresponding to a 3rd order polynomial function $\left(M_{3}\right)$

$$
f\left(n ; \boldsymbol{\theta}_{i}\right)=\theta_{i}[3] n^{3}+\theta_{i}[2] n^{2}+\theta_{i}[1] n+\theta_{i}[0] .
$$

The decreasing constraint is fulfilled introducing following inequalities on $\boldsymbol{\theta}_{i}$ :

$$
\left\{\begin{array}{l}
\theta_{i}[3]>0, \\
\theta_{i}[1]<0, \\
3 . \theta_{i}[3] N^{2}+2 . \theta_{i}[2] N+\theta_{i}[1]<0
\end{array}\right.
$$

with $N$ the length of our observation's window.
Note that if we consider the function $\mathbf{f}\left(\boldsymbol{\theta}_{i}\right)$ in the model (2) as an unconstrained straight line (i.e., $L=1$ ), the residual influence of the baseline can be modeled and canceled on the ECG.

\section{B. Model Using Piecewise Linear Function $\left(M_{\mathrm{P}}\right)$}

The decreasing part of the $T$ wave is considered as a piecewise linear function $\mathbf{f}\left(\boldsymbol{\theta}_{i}\right)$, defined as a weighted sum of a collection of functions $\mathbf{v}_{l}$ (see Fig. 2)

$$
f\left(n ; \boldsymbol{\theta}_{i}\right)=\sum_{l=1}^{L} \theta_{i}[l] v_{l}[n] .
$$

We build a collection of $L$ functions $\mathbf{v}_{l}$ which defines $L$ intervals of width $K$. We note this model as $M_{P-L} . L$ and $K$ are chosen arbitrarily such as the length $L \times K$ corresponds to the expected maximal width of the segmented decreasing part of the $\mathrm{T}$ wave. The accuracy of this knowledge is not crucial. But it has to be chosen in accordance with the trade-off between the good approximation of the $\mathrm{T}$ wave and the variance of the estimated parameters of $\mathbf{f}$ (7). Given the estimation process, increasing the number of functions will reduce the approximation error while the variance of the estimated weights increases. For all subjects, empirical values of $L$ and $K$ have been chosen equal to $3\left(M_{\mathrm{P}-3}\right)$ and 20 , respectively. The selection of these values have not been considered as the part of the global optimization.

As it will be shown in the sequel, this modeling will provide us a tractable solution that accounts for our a priori information concerning the $\mathrm{T}$ wave. So, it is expected that this knowledge reduces the bias avoiding the nonexistence of an unique solution.

In the Fig. 2, functions $\mathbf{v}_{l}$ are chosen as piecewise linear functions. In order to be consistent with the observations, some constraints are added:

1) in each interval, a negative slope is imposed for any functions combination;

2) in order to keep the continuity of the modeled $\mathrm{T}$ wave, the joining points between two consecutive intervals must respect the following constraint: the last point of the $l$ th interval must be identical to the first point of the $(l+1)$ th interval.

The aim is to build a collection of $L$ functions $\mathbf{v}_{l}$. We choose arbitrarily $L=3$ and $K=20$ as in the Fig. 2, for a sampling frequency equal to $1000 \mathrm{~Hz}$ (Note that $L$ does not influence a lot the bias of the estimator on simulated data). Therefore, three intervals are considered.

Choosing functions as in Fig. 2, on each interval, for $n \in[k \times$ $K:(k+1) \times K]$ (with $k=0, \ldots, 2)$, the $\mathrm{T}$ wave is modeled by a linear function that is a weighted sum of two non-zero functions

$$
\left\{\begin{array}{l}
f[n]=\theta_{1} \cdot v_{1}[n]+\theta_{2} \cdot v_{2}[n] ; n \in[0: K], \\
f[n]=\theta_{2} \cdot v_{2}[n]+\theta_{3} \cdot v_{3}[n] ; n \in[K: 2 K], \\
f[n]=\theta_{3} \cdot v_{3}[n] ; n \in[2 K: 3 K] .
\end{array}\right.
$$

Moreover, the $\mathrm{T}$ wave has to be modeled by a decreasing linear function, so the following conditions on each interval 
must be imposed:

$$
\left\{\begin{array}{l}
f^{\prime}[n]=\theta_{1} \cdot v_{1}^{\prime}[n]+\theta_{2} \cdot v_{2}^{\prime}[n] \leq 0 ; n \in[0: K], \\
f^{\prime}[n]=\theta_{2} \cdot v_{2}^{\prime}[n]+\theta_{3} \cdot v_{3}^{\prime}[n] \leq 0 ; n \in[K: 2 K], \\
f^{\prime}[n]=\theta_{3} \cdot v_{3}^{\prime}[n] \leq 0 ; n \in[2 K: 3 K]
\end{array}\right.
$$

where $f^{\prime}$ stands for the temporal derivative of $f$.

In order to obtain a tractable relation linking the coefficients $\theta_{i}$, the 3 functions $\mathbf{v}_{l}$ are chosen arbitrarily as

$$
\left\{\begin{array}{l}
v_{1}^{\prime}[n]<0 ; n \in[0: K], \\
v_{1}^{\prime}[n]=-v_{2}^{\prime}[n] ; n \in[0: K], \\
v_{2}^{\prime}[n]=-v_{3}^{\prime}[n] ; n \in[K: 2 K] .
\end{array}\right.
$$

These relations imply that $v_{1}$ and $v_{3}$ are decreasing respectively on the intervals $[0: K]$ and $[2 K: 3 K]$.

Imposing these properties to the functions, the conditions of continuity at the joining points ( $n=K$ and $n=2 K$ ) between two consecutive intervals need to be checked. Thus, for example when $n=K$, we get

$$
\theta_{1} v_{1}[K]+\theta_{2} v_{2}[K]=\theta_{2} v_{2}[K]+\theta_{3} v_{3}[K] .
$$

However, using (8), on each interval we obtain the relations

$$
\left\{\begin{array}{l}
v_{1}[n]=-v_{2}[n]+C_{1} ; n \in[0: K] \\
v_{2}[n]=-v_{3}[n]+C_{2} ; n \in[K: 2 K
\end{array}\right.
$$

where $C_{1}$ and $C_{2}$ are constant values.

By replacing in (9) the results (10) with $n=K$, we get

$$
\left(\theta_{2}-\theta_{1}\right) v_{2}[K]+\theta_{1} C_{1}=\left(\theta_{3}-\theta_{2}\right) v_{3}[K]+\theta_{2} C_{2} .
$$

We impose that $v_{1}[K]=v_{3}[K]=0$ which implies, given the relations (10), that $C_{1}=C_{2}$. So, the continuity for $n=K$ is ensured.

Finally, when building a collection of $L$ functions $\mathbf{v}_{l}$ such as in Fig. 2, these rules are applied:

1) the first function is decreasing on the interval $[0: K]$ and is null after;

2) the last function is null for $n \in[0:(L-2) K]$ and is decreasing on the interval $[(L-1) K: L K]$.

This implies that $\theta_{L}$ must be positive in order to keep the decreasing property of the modeled $\mathrm{T}$ wave. Besides, thanks to the hypotheses (8) and (10), the constraints on the $\theta_{i}$ s are

$$
\forall l \in[1: L-1], \theta_{i}[l]>\theta_{i}[l+1]>0 .
$$

Note that the previous development has been given without lack of generality since it is valid for any number $L$ of functions $\mathbf{v}_{l}$.

\section{RESULTS}

\section{A. Simulation Study}

The synthesized ECG during exercise using Gaussian functions is presented in Fig. 3. This ECG has constant PR intervals, and a time-varying T-P duration. This duration decreases linearly as the beat number increases. In Fig. 3, the extreme lefthand side and the extreme right-hand side $\mathrm{T}$ waves correspond respectively to the 1 st and the 400 th beat.

Theoretically, the estimated delays, i.e., the PR intervals, should be constant. It is expected that the $\mathrm{T}$ wave introduces

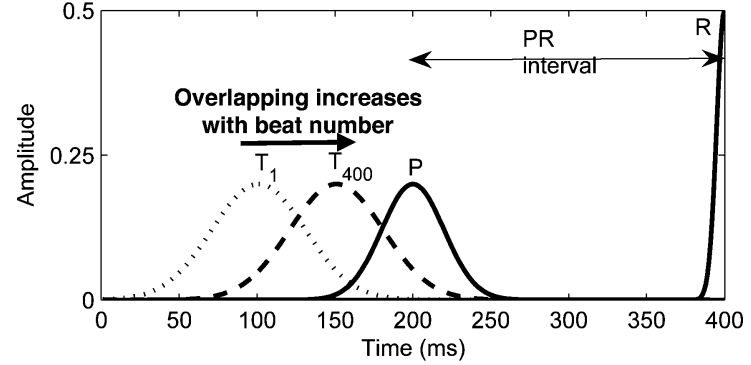

Fig. 3. Synthetic data of ECG during exercise. This ECG has constant PR intervals, and a time-varying T-P duration. This duration decreases linearly as the beat number increases. The extreme left-hand side and the extreme righthand side $\mathrm{T}$ waves correspond, respectively, to the 1st and the 400th beat.

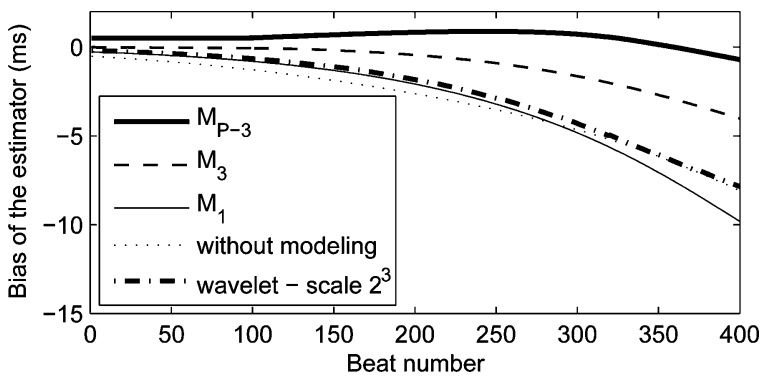

Fig. 4. Bias of the time delay estimator for the three considered models and with the wavelet technique at scale $2^{3}$ [14].

bias with a value depending on the overlapping ratio. Fig. 4 shows the time course of the bias between the real PR intervals and the estimated ones (in function of the beat number) obtained with our piecewise solution $\left(M_{\mathrm{P}}\right)$ represented with a thick solid curve. This figure also shows the bias obtained with two other models: the dashed line and the solid one correspond to a model based on a constrained third-order polynomial function $\left(M_{3}\right)$ or a decreasing single straight line $\left(M_{1}\right)$, respectively. The dotted curve corresponds to an estimation of the PR intervals without $\mathrm{T}$ wave accounting.

Whatever the chosen model, the bias is low when the T-P fusion occurs weakly. When the beat number increases, the model $M_{\mathrm{P}}$ outperforms the others. This result shows that, at least in simulation, our piecewise $\mathrm{T}$ wave model $\left(M_{\mathrm{P}}\right)$ is more accurate than the others when the overlapping appears.

Besides, Fig. 4 exhibits the bias of the estimator computed with the wavelet tool. The used wavelet is the first derivative of a Gaussian smoothing function, and the zero-crossing computation of the wavelet transform provides the location of the signal shape variation points [14]. It is known that the power spectrum of the $\mathrm{P}$ and $\mathrm{T}$ waves lie in the same range [22]. In order to avoid errors due to artifacts, the scale $2^{3}$ is chosen to detect the onset and the offset of the $\mathrm{P}$ and $\mathrm{T}$ waves as proposed in [14]. From Fig. 4, it is clear that the fusion of the two waves bias the zero-crossing location.

\section{B. Results on Real Data}

Using the proposed method of TDE, we estimate PR intervals from real ECG recorded in healthy humans during exercise and recovery. 
TABLE I

Training Status and MaXimal OXYGEn UPTAKe $\left(V_{\mathrm{O}_{2}} \mathrm{MAX}\right)$ OF STUdiEd POPULATION. SED: SEDENTARY SUBJECTS; ATH: ATHLETES

\begin{tabular}{|c|c|c|}
\hline & SED & ATH \\
\hline Hours of training/week & $<5$ & $>5$ \\
\hline$V_{\mathrm{O}_{2}} \max [\mathrm{ml} / \mathrm{min} / \mathrm{kg}]$ & $43.3 \pm 5.6$ & $68.7 \pm 6.5$ \\
\hline Age [years] & $32.5 \pm 11$ & $26.5 \pm 4.5$ \\
\hline
\end{tabular}

\section{1) Experimental Design:}

a) Subjects: Five sedentary men (SED) and seven athlete men (ATH), whose physical characteristics are shown in Table I, participated in the present study. All subjects were non-smokers, and none was taking any medication. Physical activity and alcohol and caffeinated beverages consumption were prohibited $24 \mathrm{~h}$ before any exercise testing session.

b) Protocol: All subjects performed a maximal graded exercise test. The initial load was fixed at $75 \mathrm{~W}$ for sedentary subjects or $150 \mathrm{~W}$ for athletes and increased by $37.5 \mathrm{~W}$ every 2 min until exhaustion. The pedaling rate was kept constant at 75 and $90 \mathrm{r} / \mathrm{min}$ for the SED and ATH group, respectively. During the exercise test and the preceding 5 min (rest), a one-lead ECG was recorded and digitized on-line by a 12-bit analog-to-digital converter at a sampling rate of $1000 \mathrm{~Hz}$ on a personal computer. The lead is placed collinearly to the standard DII derivation directly on the chest in order to avoid limbs motion artifacts. The DII lead is chosen because it exhibits the highest amplitude of the $\mathrm{P}$ wave. Besides, it assures the $\mathrm{T}$ wave of being positive and monophasic, and it minimizes the presence of the $U$ wave. All subjects completed the exercise test without any clinical abnormalities or discomfort.

2) Estimation of PR Intervals: Two pre-processing methods provide us the position of the $\mathrm{R}$ waves [5]. First, a threshold technique applied on the high-pass filtered and demodulated ECG, refines the estimation of the time occurrences $t_{k}$ of the $\mathrm{R}$ waves, that are roughly the $\mathrm{R}$ peaks locations. The high-pass filtering is a 500th order FIR filter designed with a hamming window and a cutoff frequency equal to $5 \mathrm{~Hz}$. Segments including each expected $\mathrm{P}$ wave and its corresponding $\mathrm{R}$ wave in sequence are formed time locked with the $t_{k}$. The length of the segments is fixed for all beats and depends of the subject. For each heart rate, the left boundary of the segment is adjusted in order to get only the decreasing part of the $\mathrm{T}$ wave, and to ensure that the whole $\mathrm{P}$ wave is encompassed (see Fig. 2). In a real case, this condition is readily achieved and the $\mathrm{T}$ wave should not be present in our observation window for low heart rates.

While the PR intervals estimator provides reliable results regarding the $\mathrm{T}$ wave overlapping, it is biased by the presence of a baseline corresponding to the respiration and other artifacts. We use the baseline removal approach proposed in [5], based on an order one polynomial substraction. Finally, we consider in the model (2) the segments $\mathbf{x}_{i}$ including each expected $\mathrm{P}$ wave and the decreasing part of the $\mathrm{T}$ wave (when both waves overlapped).

The instantaneous, or time course, of PR interval is not in the scope of this paper. Then, in order to reduce the effect of noise at the maximum exercise intensity, each set of 10 PR interval segments was replaced by the corresponding average [23], [24].

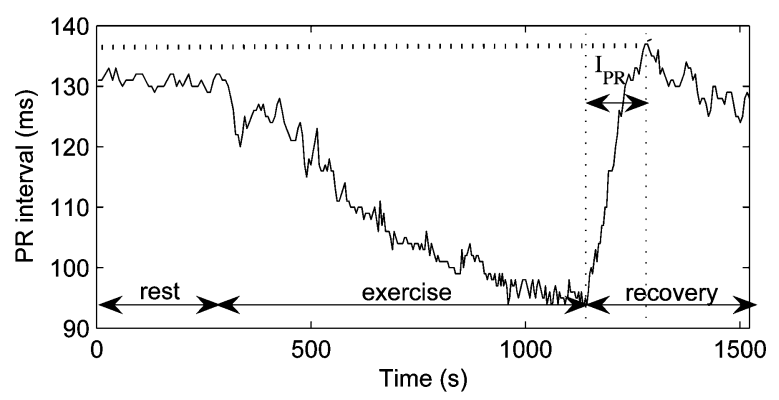

Fig. 5. Representative example of PR interval pattern in an athlete subject The $\mathrm{T}$ wave was modeled by a constrained piecewise linear function $\left(M_{\mathrm{P}}\right)$. The interval $I_{\mathrm{PR}}$ is used for the calculation of the slope $S_{\mathrm{PR}}$, indicative of the "recovery rate"; the interval $I_{\mathrm{PR}}$ is defined between the end of the exercise and the abrupt change of slope, and is delimited by the two dotted vertical lines.

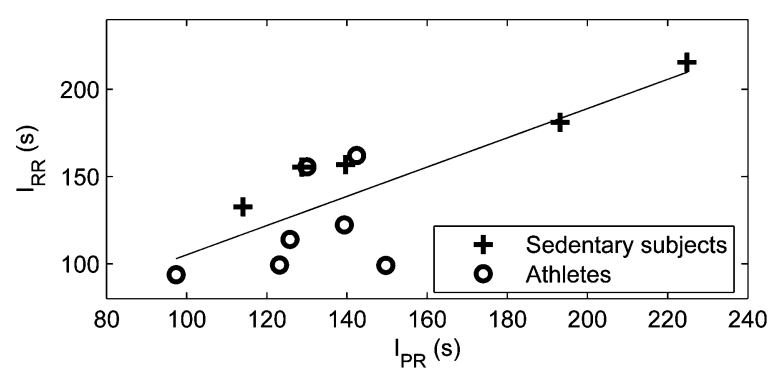

Fig. 6. Scatter diagram of the observations for the 12 subjects. Relation between the time of abrupt change of slope for PR $\left(I_{\mathrm{PR}}\right)$ and RR $\left(I_{\mathrm{RR}}\right)$ intervals $(r=0.784 ; p$-value $<0.001)$.

By computing our generalized Woody method presented in Section II, the coefficients of the model $\left(M_{1}, M_{3}\right.$, or $\left.M_{\mathrm{P}-3}\right)$ and the obtained $\widehat{d}_{i}$ s corresponding to the PR intervals up to a constant are estimated. The convergence of the algorithm is achieved after 10 iterations.

Fig. 5 shows a representative example of the PR interval pattern using the $M_{\mathrm{P}-3}$ model. The constant of the $d_{i}$ has been evaluated on the maximum of the $\mathrm{P}$ wave average, $s$, provided by the algorithm.

3) Observations During Early Recovery Phase: Consistent with our previous observations [21], [25], we show an abrupt change of PR interval slope on the early recovery phase, which is significantly correlated with the RR interval slope. This result is valid for all the models of the T wave proposed in Section III.

Fig. 6 shows the scatter diagram which characterizes the relationship between the PR and RR duration of the early recovery phase $\left(I_{\mathrm{PR}}\right.$ and $\left.I_{\mathrm{RR}}\right)$. The estimated PR intervals presented in this scatter diagram correspond to a piecewise linear function model $\left(M_{\mathrm{P}-3}\right)$. The correlation coefficients of the relation between $I_{\mathrm{PR}}$ and $I_{\mathrm{RR}}$ with different models are presented in Table II. The time occurrence of abrupt change of slope on PR and RR for all subjects are computed as follows. We consider two phases in the beginning of the recovery, each one being modeled as a straight line. The total time interval is divided into two corresponding segments. The index that splits the whole segment in two, i.e., $I_{\mathrm{PR}}$ in Fig. 5 in the case of PR intervals, minimizes the sum of mean error of the least square modeling of the two lines. 
TABLE II

CORRELATION COEFFICIENTS OF SCATTER DiAgRAMS $\left(I_{\mathrm{PR}}, I_{\mathrm{R} R}\right)$ FOR DIFFERENT T WAVE MODELS; $P$-VALUE $<0.001$

\begin{tabular}{|c|c|}
\hline T-wave model & Correlation coefficient \\
\hline$M_{P-3}$ & 0.784 \\
\hline$M_{3}$ & 0.783 \\
\hline$M_{1}$ & 0.781 \\
\hline No model & 0.275 \\
\hline
\end{tabular}

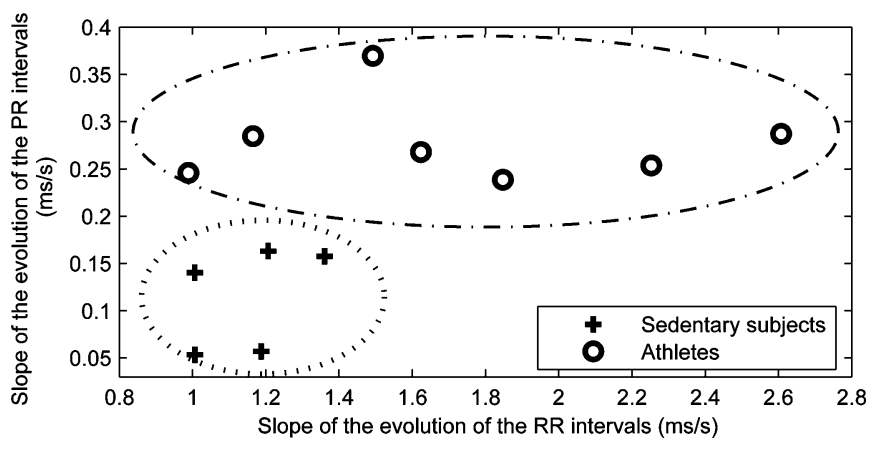

Fig. 7. Relationship between the slope of the evolutions of the PR and RR intervals for the 12 subjects. The two groups can be clustered according the PR slopes.

The time locations of these changes of slope referenced to the end of exercise, on PR and RR are related to each subject, without any effect of the training level. Additionally, the slope $S_{\mathrm{RR}}$ and $S_{\mathrm{PR}}$ of the RR and PR intervals respectively, are computed for all subjects on the time interval $I_{\mathrm{RR}}$ and $I_{\mathrm{PR}}$ bounded by the two dotted vertical lines in Fig. 5. These two bounds correspond to the end of the exercise and the time location of the change of slope respectively. This slope should convey informations relative to the "recovery rate" of the subject.

Fig. 7 relates the slopes $S_{\mathrm{PR}}$ in function of $S_{\mathrm{RR}}$ for the 12 subjects using the $M_{\mathrm{P}-3}$ model. The values of the slopes of PR are higher in ATH than in SED subjects. A k-means clustering algorithm has been applied on our data set assuming a cluster for the SED and an other one for the ATH. While exploiting data of both PR and RR intervals, we obtain $33 \%$ of misclassification, $60 \%$ considering the data of the RR intervals only and $0 \%$ considering the data of the PR intervals only.

Similar results (with less resolution however) are obtained using the single straight line $\left(M_{1}\right)$ or the third-order polynomial function $\left(M_{3}\right)$ models. To compare the different models with a number of cluster equal to 2 , we compute the statistic of the Welch's test, $t$, and a k-means criterion, $C$, defined as

$$
C=\frac{\text { distance between the two centroids }}{\text { sum of the two radius }} \text {. }
$$

Raw PR intervals indexes (without modeling improvement) are not reported because of the $25 \%$ of misclassification of the k-means algorithm. For the three models, the Welch's test is positive with a $p$-value $<0.001$. The criterion $C$ for the three models is presented in Table III. The highest value is provided by the $M_{\mathrm{P}}$ model meaning that the best clustering is reached for this model.

4) $P R / R R$ Hysteresis Phenomenon: Previous studies on ECG recordings under exercise conditions show that there
TABLE III

Criterion $C$ OF K-MEANS Algorithm DeFined on (13) FOR DIFFERENT MODELS

\begin{tabular}{|c|c|}
\hline T-wave model & $C$ \\
\hline$M_{P-3}$ & 0.527 \\
\hline$M_{3}$ & 0.465 \\
\hline$M_{1}$ & 0.414 \\
\hline
\end{tabular}

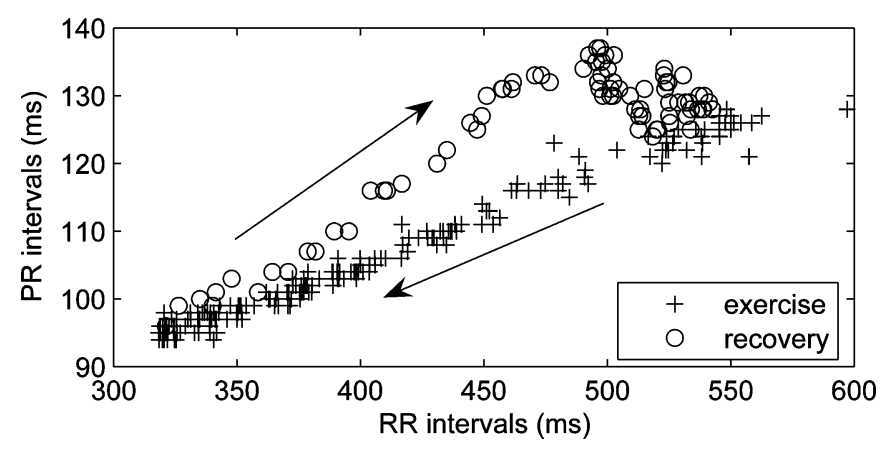

Fig. 8. Evolutionof PR intervals $\left(M_{\mathrm{P}-3}\right.$ model) in function of RR intervals during exercise $(+)$ and recovery $(\circ)$. We note a clockwise hysteresis phenomenon.

TABLE IV

MEAN AND STANDARD DEVIATION OF HYSTERESIS CRITERION FOR DIFFERENTLY TRAINED ATHLETES GROUPS AND DIFFERENT T-WAVE MODELS

\begin{tabular}{|c|c|c|}
\hline T-wave model & SED & ATH \\
\hline$M_{P-3}$ & $7.84 \pm 2.52$ & $13.49 \pm 3.64$ \\
\hline$M_{3}$ & $6.33 \pm 4.32$ & $13.35 \pm 2.58$ \\
\hline$M_{1}$ & $7.05 \pm 3.12$ & $9.70 \pm 8.74$ \\
\hline
\end{tabular}

exists a nonlinear relationship between PR and RR intervals which exhibits an hysteresis shape, [4], [5].

Fig. 8 shows a representative example of the PR versus RR interval relationship in one athlete. PR intervals were estimated with the piecewise linear function model $\left(M_{\mathrm{P}-3}\right)$. Note that for a same value of RR interval in exercise and recovery, the PR interval is increased during the recovery.

To quantify the presence of an hysteresis phenomenon, we calculated an hysteresis criterion defined as the difference of areas between the recovery curve and the exercise curve, normalized by the range of RR intervals of each subject. The study of this criterion reveals the existence of the hysteresis phenomenon for all subjects. The mean and the standard deviation of this criterion for all T-wave models are presented in Table IV for the two groups of subjects. As in the previous Section IV-B3, the two groups of subjects are identified according the mean of the hysteresis criterion: the SED and the ATH. Using a Welch's test, we find that the averaged hysteresis criterion is significantly different between groups $(p$-value $<0.001)$. We also note that subjects with the more pronounced early recovery PR slope have a substantially greater hysteresis.

\section{DISCUSSION}

Our findings based on real ECG data showed that: 1) the PR/RR interval relationship during exercise and its recovery demonstrates an hysteresis pattern; 2) this hysteresis phenomenon tends to be more pronounced in athletes versus 
nonathletes; and 3) the slope of PR return toward control values during the early recovery phase tends to be more accentuated in athletes versus nonathletes.

The atrioventricular (AV) and sinus nodes activity is tightly regulated by the autonomic nervous system to match the organism demand (metabolic in particular). Despite that autonomic stimulation tends to produce similar changes in the heart period and AV conduction time (i.e., lengthen and shorten with vagal and sympathetic stimulation, respectively), it has been demonstrated that the net autonomic effect on the AV and sinus nodes are independent of each other [26], [27]. For instance, Kowallick et al. showed in healthy subjects that sudden spontaneous increases in heart rate with change in position during sleep, are associated with either a concomitant shortening, no change or a lengthening of PR interval. Similarly, using pharmacological blockade in patients with implanted dual-chamber devices, Kannankeril et al. found poor correlation among the parasympathetic effects on the AV and sinus nodes during exercise and recovery. This data are consistent with the previous studies [28], [29] which demonstrated that the neural circuitry which innervates the AV and the sinus nodes is specific and independent. Based on these findings, we speculate that a greater parasympathetic re-engagement at the AV versus sinus node level would explain the hysteresis in the PR-RR relationship, when data from exercise and recovery are compared.

Moreover, the vagal mediated-recovery in heart rate (sinus node level) has been shown to occur more rapidly in athletes compared to non-athletes [30], [31]. We make the assumption that vagal re-engagement at the AV level is also more accentuated in athletes compared to nonathletes. This would explain the greater hysteresis and the steeper PR slope observed in athletes during early recovery phase. Further studies are required to verify these different assumptions. Besides, the age influence should be checked on a larger dataset.

\section{CONCLUSION}

The problem of time delay estimation for noisy and unknown signals is considered. The generalization of the observation model is proposed and permits us to address more difficult TDE problems in electrocardiographic signal processing. In particular, a focus is made on the TDE method applied to the PR intervals estimation in ECG recorded during exercise. This topic is particulary difficult as the T-P fusion occurs during high heart rate in exercise. Several models are also proposed for the $\mathrm{T}$ wave in order to reduce its influence on the estimation of the PR intervals. The feasibility of the proposed technique is corroborated by the results on synthetic data and finally on real ECG recorded in exercise. Furthermore, the proposed TDE method can be used for many other problems in biomedical signal processing, and not necessarily in the electrocardiographic area. Actually, the proposed generalization can be used for any time delay estimation problem where possible disturbance can be modeled, excluding the proposed PR intervals estimation in exercise. This new tool can be adapted for QT interval estimation in ECG recorded in exercise whilst taking into account the $\mathrm{P}$ wave in this instance.
Results on synthetic data or on real ECG recorded in exercise exhibit better performances when the PR interval estimation is computed by combining a piecewise linear function model for the $\mathrm{T}$ wave and our generalized Woody method. The introduction of the decreasing requirement is due to a need to reduce the variance in the estimation process. The corresponding constraints have a cost: it is assumed that the $\mathrm{T}$ wave is observed in its decreasing part. That is met using adapted derivation leads and a matched window. A refinement to a fixed window definition would be to adapt its position using the Bazett correction or any Q-T predictor [32], [33].

Applications of the PR intervals estimation on real ECG recordings on sedentary and athlete subjects lead to many results: 1) the subjects can be characterized according to their training level studying the PR slope in the early recovery phase; and 2) an hysteresis phenomenon exists in the relation PR-RR when data from exercise and its recovery are compared.

The problem of estimating heart periods and consequently the problem of understanding the autonomic nervous system activity during exercise and recovery is still of interest since it would contribute to improve performances of cardiac pacemakers by optimizing the programming of these pacing systems.

\section{APPENDIX}

Considering the observation model defined by (2), the iterative MLE is used in order to estimate the delays $d_{i}$.

The noise $e_{i}(n)$ is assume to be white Gaussian with null mean and variance $\sigma^{2}$. Then, the probability of $x_{i}$ at time sample $n$, given $s(n), \boldsymbol{\theta}_{i}, \alpha_{i}$ and $d_{i}$, can be expressed with

$$
\begin{aligned}
p( & \left.x_{i}(n) ; s(n), d_{i}, \boldsymbol{\theta}_{i}, \alpha_{i}\right) \\
& =\frac{1}{\sigma \sqrt{2 \pi}} \exp \left(-\frac{1}{2 \sigma^{2}}\left(x_{i}(n)-f\left(n ; \boldsymbol{\theta}_{i}\right)-\alpha_{i} s_{d_{i}}(n)\right)^{2}\right) .
\end{aligned}
$$

Since the noise at different instants is independent, so are the observations. Then, for $\mathbf{x}_{i}=\left[x_{i}(1), x_{i}(2), \ldots, x_{i}(N)\right]^{T}$ where $N$ denotes the number of data samples for each trial

$$
p\left(\mathbf{x}_{i}\right)=\prod_{n=1}^{N} p\left(x_{i}(n)\right) .
$$

Thus,

$$
\begin{aligned}
& p\left(\mathbf{x}_{i} ; \mathbf{s}, d_{i}, \boldsymbol{\theta}_{i}, \alpha_{i}\right) \\
& \left.=\frac{1}{\left(2 \pi \sigma^{2}\right)^{\frac{N}{2}}} \exp \left(-\frac{1}{2 \sigma^{2}} \sum_{n=1}^{N}\left(x_{i}(n)-f\left(n ; \boldsymbol{\theta}_{i}\right)\right)-\alpha_{i} s_{d_{i}}(n)\right)^{2}\right) .
\end{aligned}
$$

Then, for all $i$, the pdf of the processes $\mathbf{x}_{i} \mathbf{s}$, given the delay $d_{i}$ s, the coefficients $\boldsymbol{\theta}_{i}$, the parameter of amplitude jitter $\alpha_{i}$ and signal vectors $\mathbf{s}$, is

$$
\begin{aligned}
p\left(\mathbf{X} ; \mathbf{s}, \mathbf{d}, \boldsymbol{\theta}_{i}, \alpha_{i}\right)= & \frac{1}{\left(2 \pi \sigma^{2}\right)^{\frac{N I}{2}}} \exp \left(-\frac{1}{2 \sigma^{2}} \sum_{i=1}^{I} \sum_{n=1}^{N}\left(x_{i}(n)\right.\right. \\
& \left.\left.-f\left(n ; \boldsymbol{\theta}_{i}\right)-\alpha_{i} s_{d_{i}}(n)\right)^{2}\right)
\end{aligned}
$$


where $\mathbf{X}=\left[\mathbf{x}_{1}, \mathbf{x}_{2}, \ldots, \mathbf{x}_{I}\right], \mathbf{s}=\left[s_{1} s_{2}, \ldots, s_{I}\right]^{T}$, and $\mathbf{d}=$ $\left[d_{1} d_{2}, \ldots, d_{I}\right]^{T}$.

The objective is to estimate the $d_{i} \mathrm{~s}$ for all $i$, in other words to maximize $p\left(\mathbf{X} ; \mathbf{s}, \mathbf{d}, \boldsymbol{\theta}_{i}, \alpha_{i}\right)$. So, according the MLE, the criterion $J$ to be minimized is

$$
J=\sum_{i=1}^{I}\left\|\mathbf{x}_{i}-\alpha_{i} \mathbf{s}_{d_{i}}-\mathbf{f}\left(\boldsymbol{\theta}_{i}\right)\right\|^{2}
$$

where $\mathbf{f}\left(\boldsymbol{\theta}_{i}\right)=\left[f\left(1 ; \boldsymbol{\theta}_{i}\right), f\left(2 ; \boldsymbol{\theta}_{i}\right), \ldots, f\left(N ; \boldsymbol{\theta}_{i}\right)\right]^{T}$, where $N$ denotes the number of data samples for each trial. To solve this kind of problem, first we make a change of variables

$$
\mathbf{y}_{i}=\mathbf{x}_{i}-\mathbf{f}\left(\boldsymbol{\theta}_{i}\right)
$$

So, we consider the criterion

$$
J=\sum_{i=1}^{I}\left\|\mathbf{y}_{i}-\alpha_{i} \mathbf{s}_{d_{i}}\right\|^{2}=\sum_{i=1}^{I}\left(\mathbf{y}_{i}^{T} \mathbf{y}_{i}+\alpha_{i}^{2} \mathbf{s}_{d_{i}}^{T} \mathbf{s}_{d_{i}}-2 \alpha_{i} \mathbf{y}_{i}^{T} \mathbf{s}_{d_{i}}\right) .
$$

The noise sweeps $e_{i}(n)$ are modeled as trials of a common zero mean stationary Gaussian process and we assume that $e_{j}$ and $e_{k}$ are independent for $j \neq k$. Since the signal $s(n)$ is unknown and has a bounded support, the properties $\mathbf{s}_{d_{i}}^{T} \mathbf{s}_{d_{i}}=\mathbf{s}^{T} \mathbf{s}$ and $\mathbf{y}_{i}^{T} \mathbf{s}_{d_{i}}=\mathbf{y}_{i,-d_{i}}^{T} \mathbf{s}$ are valid. Note that it could be proven in the continuous time domain assuming that $\mathbf{s}$ is not truncated in the observation window for any values of $d_{i}$. The minimization of the criterion (20) with respect to $s(n)$ is then

$$
\widehat{\mathbf{s}}=\frac{1}{I} \sum_{k=1}^{I} \frac{1}{\alpha_{k}} \mathbf{y}_{k,-d_{k}}
$$

Substituting this estimation for $\mathbf{s}$ in (20), we obtain

$$
\begin{aligned}
J & =\sum_{i=1}^{I}\left\|\mathbf{y}_{i}-\alpha_{i} \widehat{\mathbf{s}}_{d_{i}}\right\|^{2} \\
& =\sum_{i=1}^{I}\left\|\mathbf{y}_{i}-\frac{\alpha_{i}}{I} \sum_{k=1}^{I} \frac{1}{\alpha_{k}} \mathbf{y}_{k, d_{i}-d_{k}}\right\|^{2} \\
& =\sum_{i=1}^{I}\left\|\mathbf{y}_{i}-\frac{\alpha_{i}}{I} \sum_{k=1}^{I} \frac{1}{\alpha_{k}}\left(\mathbf{x}_{k, d_{i}-d_{k}}-\mathbf{f}_{d_{i}-d_{k}}\left(\boldsymbol{\theta}_{k}\right)\right)\right\|^{2} .
\end{aligned}
$$

Using the equation (19), we obtain the criterion to minimize

$$
J=\sum_{i=1}^{I}\left\|\mathbf{x}_{i}-\mathbf{f}\left(\boldsymbol{\theta}_{i}\right)-\frac{\alpha_{i}}{I} \sum_{k=1}^{I} \frac{1}{\alpha_{k}}\left(\mathbf{x}_{k, d_{i}-d_{k}}-\mathbf{f}_{d_{i}-d_{k}}\left(\boldsymbol{\theta}_{k}\right)\right)\right\|^{2}
$$

Developing (22), we get

$$
\begin{aligned}
J=\| \mathbf{x}_{1}-\mathbf{f}\left(\boldsymbol{\theta}_{1}\right)-\frac{\alpha_{1}}{I}\left(\frac{1}{\alpha_{1}}\left(\mathbf{x}_{1,0}-\mathbf{f}\left(\boldsymbol{\theta}_{1}\right)\right)\right. \\
\left.\quad+\frac{1}{\alpha_{2}}\left(\mathbf{x}_{2, d_{1}-d_{2}}-\mathbf{f}_{d_{1}-d_{2}}\left(\boldsymbol{\theta}_{2}\right)\right)+\cdots\right) \|^{2}+\cdots
\end{aligned}
$$

$$
\begin{aligned}
& +\| \mathbf{x}_{i}-\mathbf{f}\left(\boldsymbol{\theta}_{i}\right)-\frac{\alpha_{i}}{I}\left(\frac{1}{\alpha_{1}}\left(\mathbf{x}_{1, d_{i}-d_{1}}-\mathbf{f}_{d_{i}-d_{1}}\left(\boldsymbol{\theta}_{1}\right)\right)\right. \\
& \left.+\frac{1}{\alpha_{2}}\left(\mathbf{x}_{i, d_{i}-d_{2}}-\mathbf{f}_{d_{i}-d_{2}}\left(\boldsymbol{\theta}_{2}\right)\right)+\cdots\right) \|^{2}+\cdots
\end{aligned}
$$

We observe that $d_{i}$ appears especially in the $i$ th term and is present only once in the others terms. Then, we can make the approximation that in the others terms the $d_{i}$ s influence is negligible if we consider that $I$ is large enough. This global minimization can be split in a sum of functions $J_{i}$ to be minimized

$$
J_{i}=\left\|\mathbf{x}_{i}-\mathbf{f}\left(\boldsymbol{\theta}_{i}\right)-\frac{\alpha_{i}}{I} \sum_{k=1}^{I} \frac{1}{\alpha_{k}}\left(\mathbf{x}_{k, d_{i}-d_{k}}-\mathbf{f}_{d_{i}-d_{k}}\left(\boldsymbol{\theta}_{k}\right)\right)\right\|^{2} .
$$

This global minimization will be performed in an iterative manner as in [10].

\section{REFERENCES}

[1] R. Shouldice, C. Heneghan, P. Nolan, and P. Nolan, "PR and PP ECG intervals as indicators of autonomic nervous innervation of the cardiac sinoatrial and atrioventricular nodes," in Proc. IEEE EMBS Conf. Neural Eng., 2003, pp. 261-264.

[2] Task Force of the European Society of Cardiology the North American Society of Pacing Electrophysiology, "Heart rate variability: Standards of measurement, physiological interpretation, and clinical use," Circulation, vol. 93, pp. 1043-1065, 1996.

[3] J. Froning and V. Froelicher, "Detection and measurement of the P-wave and T-wave during exercise testing using combined heuristic and statistical methods," J. Electrocardiol., vol. 20, pp. 146-156, 1987.

[4] A. Cabasson, O. Meste, G. Blain, and S. Bermon, "A new method for the PP-PR hysteresis phenomenon enhancement under exercise conditions," Comput. Cardiol., vol. 32, pp. 723-726, 2005.

[5] O. Meste, G. Blain, and S. Bermon, "Hysteresis analysis of the PRPPrelation under exercise conditions," Comput. Cardiol., vol. 31, pp. 461464, 2004.

[6] C. H. Knapp and G. C. Carter, "The generalized correlation method for estimation of time delay," IEEE Trans. Acoust., Speech, Signal Process., vol. 24, no. 4, pp. 320-327, Aug. 1976.

[7] R. Boucher and J. Hassab, "Analysis of discrete implementation of generalized cross-correlator," IEEE Trans. Acoust. Speech, Signal Process., vol. ASSP-29, no. 3, pp. 609-611, Jun. 1981.

[8] G. Carter, "Coherence and time delay estimation," Proc. IEEE, vol. 75, no. 2, pp. 236-255, Feb. 1987.

[9] C. D. Woody, "Characterization of an adaptative filter for the analysis of variable latency neuroelectric signals," Med. Biol. Eng. Comput., vol. 5, pp. 539-553, 1967.

[10] A. Cabasson and O. Meste, "Time delay estimation: A new insight into the Woody's method," IEEE Signal Process. Lett., vol. 15, pp. 573-576, 2008.

[11] L. Xu, P. Stoica, J. Li, S. L. Bressler, X. Shao, and M. Ding, "ASEO: A method for the simultaneous estimation of single-trial event-related potentials and ongoing brain activities," IEEE Trans. Biomed. Eng., vol. 56, no. 1, pp. 111-121, Jan. 2009.

[12] D. T. Pham, J. Möcks, W. Köhler, and T. Gasser, "Variable latencies of noisy signals: Estimation and testing in brain potential data," Biometrika, vol. 74, pp. 525-533, 1987.

[13] P. Jaśkowski and R. Verleger, "Amplitudes and latencies of singletrial ERP's estimated by a maximum-likelihood method," IEEE Trans. Biomed. Eng., vol. 46, no. 8, pp. 987-993, Aug. 1999.

[14] J. Sahambi, S. Tandon, and R. Bhatt, "Using wavelet transforms for ECG characterization-An on-line digital signal processing system," IEEE Eng. Med. Biol. Mag., vol. 16, no. 1, pp. 77-83, Jan./Feb. 1997.

[15] T. Gasser, W. Köhler, C. Jennen-Steinmetz, and L. Sroka, "The analysis of noisy signals by nonparametric smoothing and differentiation," IEEE Trans. Biomed. Eng., vol. BME-33, no. 12, pp. 1129-1133, Dec. 1986. 
[16] T. Gasser, J. Möcks, W. Köhler, and J. De Weerd, "Performance and measures of performances for estimators of brain potentials using a real data," IEEE Trans. Biomed. Eng., vol. BME-33, no. 10, pp. 949-956, Oct. 1986.

[17] J. Forester, H. Bo, J. Sleigh, and J. Henderson, "Variability of R-R,P waveto-R wave, and R wave-to-T wave intervals," Amer. J. Physiol., vol. 248, pp. H2857-H2860, 1999.

[18] S. Boudaoud, H. Rix, and O. Meste, "Curve registration for study of pwave morphing during exercise," Comput. Cardiol., vol. 31, pp. 433-436, 2004.

[19] P. Stoica and J. Li, "On nonexistence of the maximum likelihood estimate on blind multichannel identification," IEEE Signal Process. Mag., vol. 21, no. 4, pp. 99-101, Jul. 2005.

[20] C. L. Lawson and R. J. Hanson, Solving Least Squares Problems. Englewood Cliffs, NJ: Prentice-Hall, 1974.

[21] A. Cabasson, O. Meste, G. Blain, and S. Bermon, "Estimation, analysis and comparison of the PR and RR intervals under exercise conditions and recovery," Comput. Cardiol., vol. 33, pp. 497-500, 2006.

[22] N. Thakor, J. Webstor, and W. Thompkins, "Estimation of the QRS complex power spectra for design of a QRS filter," IEEE Trans. Biomed. Eng., vol. BME-31, no. 11, pp. 702-706, Nov. 1984

[23] M. Evanich, O. Newberry, and L. Patridge, "Some limitations of periodic noise removal by averaging techniques," J. Appl. Physiol., vol. 33, pp. 536-541, 1972.

[24] H. H. Ros, A. S. M. Koeleman, and T. J. Akker, "The technique of signal averaging and its practical application in the separation of atrial and HisPurkinje activity," in Signal Averaging Technique in Clinical Cardiology: International Symposium, Cologne, V. Hombach and H. H. Hilger, Eds. May 7-9, 1981. Stuttgart/New York, Schattauer Verlag, pp. 3-14.

[25] A. Cabasson, O. Meste, G. Blain, and S. Bermon, "A new modeling of the overlapping $\mathrm{T}$ wave for the efficient estimation of the P-R intervals during exercise and recovery," in Proc. IEEE Eng. Med. Biol. Conf., 2007, vol. 29 , pp. 616-619.

[26] P. Kowallick and M. Meesmann, "Independent autonomic modulation of the human sinus and AVnodes: Evidence from beat-to-beat measurements of PRand PP intervals during sleep," J. Cardiovasc. Electrophysiol., vol. 6, no. 11, pp. 993-1003, 1995.

[27] P. J. Kannankeril and J. J. Goldberger, "Parasympathetic effects on cardiac electrophysiology during exercise and recovery," Amer. J. Physiol. Heart Circ. Physiol., vol. 282, pp. H2091-H2098, 2002.

[28] A. D'Avila, M. Scanavacca, E. Sosa, J. Ruskin, and V. Y. Reddy, "Pericardial anatomy for the interventional electrophysiologist," J. Cardiovasc. Electrophysiol., vol. 14, pp. 422-430, 2003.

[29] H. Inoue and D. Zipes, "Changes in atrial and ventricular refractoriness and in atrioventricular nodal conduction produced by combinations of vagal and sympathetic stimulation that result in a constant spontaneous sinus cycle length," Circ. Res., vol. 60, pp. 942-951, 1987.

[30] K. Imai, H. Sato, M. Hori, H. Kusuoka, H. Ozaki, H. Yokoyama, H. Takeda, M. Inoue, and T. Kamada, "Vagally mediated heart rate recovery after exercise is accelerated in athletes but blunted in patients with chronic heart failure," Amer. J. Coll. Cardiol., vol. 24, pp. 1529-1535, 1994.

[31] J. Sugawara, H. Murakami, S. Maeda, S. Kuno, and M. Matsuda, "Change in post-exercise vagal reactivation with exercise training and detraining in young men,” Eur. J. Appl. Physiol., vol. 85, pp. 259-263, 2001.

[32] P. Rautaharju, J. Warren, and H. Calhoun, "Estimation of QT prolongation. A persistent, avoidable error in computer electrocardiography," $J$. Electrocardiol., vol. 23, pp. 111-117, 1990.

[33] K. Aytemir, N. Maarouf, M. Gallagher, Y. Yap, J. Waktare, and M. Malik, "Comparison of formulae for heart rate correction of QT interval in exercise electrocardiograms," Pacing Clin. Electrophysiol., vol. 22, no. 9, pp. 1397-1401, 1999.

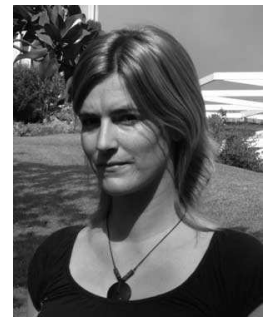

Aline Cabasson (S'05) was born in Fréjus, France, in 1981. She received the M.Sc. degree in electronic engineering from the Engineering School Polytech'Nice, Sophia Antipolis, France, and the M.Sc. degree in signal processing and communication, and the Ph.D. degree in automatic, image and signal processing from the University of Nice, Sophia Antipolis, in 2005 and 2008, respectively.

She is currently with the Laboratory I3S, Centre National de la Recherche Scientifique, University of Nice. Her research interests include biomedical signal processing, especially in the analysis of the electrocardiograms.

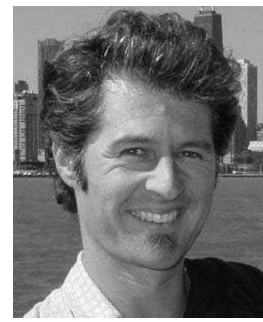

Olivier Meste (M'06) received the M.Sc. degree in automatic and signal processing, and the Ph.D. degree in scientific engineering from the University of Nice, Sophia Antipolis, France, in 1989 and 1992, respectively.

He was with the École Supérieure en Sciences Informatiques, Nice, as an Assistant Professor. He is currently a Professor at the Laboratory I3S, Centre National de la Recherche Scientifique, University of Nice. He is a Researcher at the Biomed group of the I3S Laboratory, where his research interests are in digital processing, time-frequency representations, and modeling to biological signals and systems.

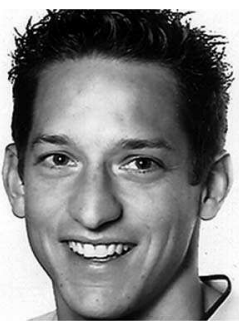

Grégory Blain received the M.Sc. and the Ph.D degrees in sports sciences from the University of Aix-Marseille II, Aix-Marseille, France, in 2001 and 2004, respectively.

$\mathrm{He}$ is currently a Research Associate at the Laboratory of Physiological Adaptations, Motor Performance and Health, Faculty of Sports Sciences, Nice. His research interests include cardiorespiratory and exercise physiology in humans.

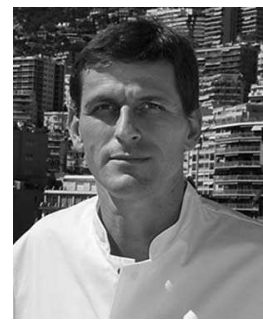

Stéphane Bermon received the M.D. degree from Nice University Medical School, Nice, France, and the $\mathrm{Ph} . \mathrm{D}$. degree in exercise physiology from the University of Aix-Marseille II, Marseille, and a specialized Master degree in health engineering from the École Centrale de Paris.

Since 2006, he is a sports physician and exercise physiologist at the Institute of Sports Medicine and Surgery, Monte Carlo, Monaco. He is the author and coauthor of more than 25 books or peer-reviewed scientific papers.

Dr. Bermon is a Member of the Medical and Antidoping Commission of the International Association of Athletics Federations. 